\title{
PP13
}

\section{FUTURE PHOTOMETRY BASED ON SOLID-STATE LIGHTING PRODUCTS}

\author{
Tuomas Poikonen et al.
}

DOI 10.25039/x46.2019.PP13

from

CIE x046:2019

Proceedings

of the

29th CIE SESSION

Washington D.C., USA, June 14 - 22, 2019

(DOI 10.25039/x46.2019)

The paper has been presented at the 29th CIE Session, Washington D.C., USA, June 14-22, 2019. It has not been peer-reviewed by CIE.

(C) CIE 2019

All rights reserved. Unless otherwise specified, no part of this publication may be reproduced or utilized in any form or by any means, electronic or mechanical, including photocopying and microfilm, without permission in writing from CIE Central Bureau at the address below. Any mention of organizations or products does not imply endorsement by the CIE.

This paper is made available open access for individual use. However, in all other cases all rights are reserved unless explicit permission is sought from and given by the CIE.

CIE Central Bureau

Babenbergerstrasse 9

A-1010 Vienna

Austria

Tel.: +4317143187

e-mail: ciecb@cie.co.at

www.cie.co.at 


\title{
FUTURE PHOTOMETRY BASED ON SOLID-STATE LIGHTING PRODUCTS
}

\author{
Poikonen, T. ${ }^{1}$, Pulli, T. ${ }^{2}$, Kokka, A. ${ }^{2}$, Askola, J²., Dönsberg, T. ${ }^{1,2}$, Šmíd, M. ${ }^{3}$, Kliment, P. ${ }^{3}$, \\ Pons, A. ${ }^{4}$, Ferrero, A. ${ }^{4}$, Kübarsepp, T. ${ }^{5}$, Gál, P. ${ }^{6}$, Gerloff, T.7, Sperling, A. ${ }^{7}$, Källberg, S. ${ }^{8}$, \\ Dekker, P. ${ }^{9}$, Thorseth, A. ${ }^{10}$, Jost, S. ${ }^{11}$, Klej, A. ${ }^{12}$, Brida, G. ${ }^{13}$, Reiners, T. ${ }^{14}$, \\ Blattner, P. ${ }^{15}$, Stuker, F. ${ }^{15}$, Ludwig, K. ${ }^{16}$, Schneider, M. ${ }^{17}$ \\ ${ }^{1}$ VTT, Espoo, FINLAND, ${ }^{2}$ Aalto University, Espoo, FINLAND, ${ }^{3}$ ČMI, Prague, CZECH \\ REPUBLIC, ${ }^{4}$ CSIC, Madrid, SPAIN, ${ }^{5}$ Metrosert, Tallinn, ESTONIA, ${ }^{6}$ BFKH, Budapest, \\ HUNGARY, 7 PTB, Braunschweig, GERMANY, ${ }^{8}$ RISE, Borås, SWEDEN, ${ }^{9}$ VSL, Delft, \\ NETHERLANDS, ${ }^{10}$ DTU, Rosskilde, DENMARK, ${ }^{11}$ ENTPE, Lyon, FRANCE, ${ }^{12}$ Signify, \\ Eindhoven, NETHERLANDS, ${ }^{13}$ INRIM, Turin, ITALY, ${ }^{14}$ LMT, Berlin, GERMANY, \\ ${ }^{15}$ METAS, Bern-Wabern, SWITZERLAND, ${ }^{16}$ OSRAM, Augsburg, GERMANY, \\ 17 OSRAM OS, Regensburg, GERMANY \\ tuomas.poikonen@vtt.fi
}

\begin{abstract}
Research project "Future photometry based on solid-state lighting products" (EMPIR 15SIB07 PhotoLED) has investigated the fundamental requirements for photometry based on white lightemitting diode (LED) sources. The project has developed new LED illuminants, LED standard lamps for luminous intensity and luminous flux, as well as new photometric measurement methods, addressing many technical challenges of the CIE research strategy. In this paper, we present the outcome of the 3-year scientific research project, whose work has been carried out by NMIs, universities, test laboratories and industrial partners working in the field of photometry and solid-state lighting.
\end{abstract}

Keywords: photometry, colorimetry, LED, illuminant, reference spectrum, luminous intensity, luminous flux, photometer, PQED, calibration, fisheye camera, integrating sphere

\section{Background}

For a century, incandescent standard lamps have been used by national metrology institutes (NMIs) and other laboratories as the reference light sources [CIE 2002] in calibrations of $V(\lambda)$ filtered illuminance and luminance meters [ISO/CIE 2014], integrating sphere photometers and goniophotometers. Many laboratories rely on source-based photometric scales that have been established using a large number of standard lamps with well-known ageing characteristics. Many of these scientific grade light sources were developed and manufactured by companies who produced tungsten filament lamps among other lighting products for the consumer market. Phasing out of consumer tungsten filament lamps may have a great influence on the availability of scientific grade incandescent lamps in the future. Therefore, it is important to investigate alternative technologies as well as requirements for manufacturing new scientific grade standard lamps for maintenance and transfer of the units of luminous intensity and luminous flux between laboratories, and for international key-comparisons in photometry.

\section{Methods and results}

\subsection{LED illuminants and reference spectrum}

The new LED-based photometric system requires a new LED reference spectrum for calibration use, similar to the CIE Standard Illuminant $A$ that is used as the reference spectrum for incandescent calibration sources in luminous responsivity calibrations of photometers [CIE 2004]. Calibrating a photometer against LED calibration source with a commonly agreed spectrum will lead to reduced spectral mismatch errors, if the photometer is used for measuring similar type of light. Currently, photometers used in laboratories and in the field are calibrated using the CIE Standard Illuminant A spectrum, although more and more LED light is measured. As in the case of Standard Illuminant $A$, it is beneficial to link the reference spectrum between 
photometry and colorimetry, to enable colorimetric calculations with the spectrum used for calibration of photometers for general light measurements.

The research started by investigating representative LED spectra and possible new LED illuminants. A total of 1500 spectral power distributions of white LED products were measured and collected from the project partners and stakeholders for the development of new LED illuminants. The white LED spectra that were based on phosphor-converted blue LEDs (BL) were categorised according to their correlated colour temperatures (CCTs) into 8 different bins between $2700 \mathrm{~K}$ and $6500 \mathrm{~K}$ according to standard ANSI C78.377-2011. In addition, 4 special shapes including red, green, blue (RGB), phosphor-converted blue LEDs with red LEDs (hybrid) and phosphor-converted ultraviolet LEDs (VLED) were chosen as potential LED illuminants for colorimetry. The analysis of the LED illuminants has been described in [JOST et al. 2017]. The spectral data were shared with CIE TC1-85 to give input on the Technical Report CIE15: Colorimetry, $4^{\text {th }}$ edition [CIE 2018]. The LED illuminants developed in the project are shown in Figure 1.

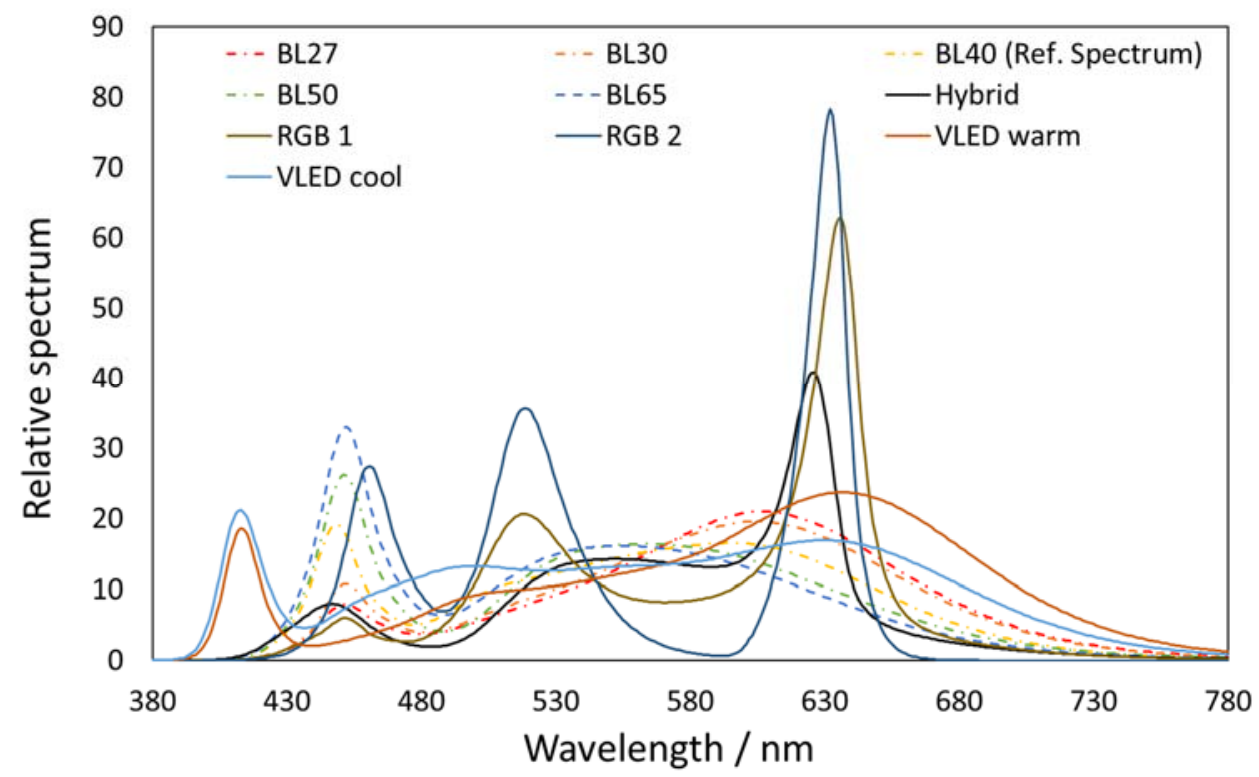

Figure 1 - Spectral power distributions of the new LED illuminants developed in the project.

In order to identify the optimal spectral power distribution of an LED source for calibration use, extensive spectral mismatch analysis was carried out using a Monte Carlo simulation, calculating spectral mismatch errors for the measurements of the 1500 LED products with spectral responsivity data of over 100 photometers, using the phosphor converted white LED illuminants as the potential reference spectra [Kokka 2018]. The results show that a single LED reference spectrum with CCT close to $4100 \mathrm{~K}$ would lead to the smallest mean of absolute errors (MAE) in spectral mismatch, when measuring LED products of different types, reducing spectral errors by a factor of two on average compared to using an incandescent source with the CIE Standard Illuminant A spectrum for calibration of the photometers (Figure 2, left). 

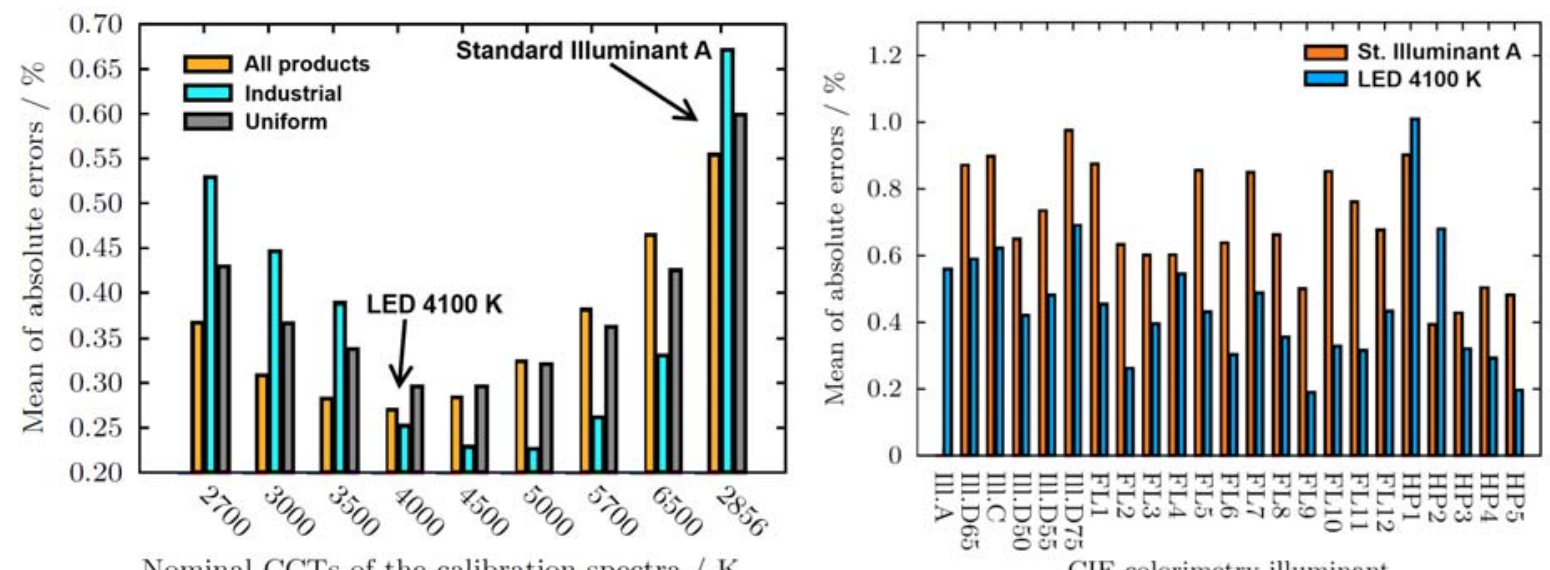

Figure 2 - Mean absolute spectral mismatch errors obtained in measurements of LED lighting, when calibrating photometers with LED sources having difference CCTs (left) and in the case of measuring other types of light represented by the illuminants of CIE15: Colorimetry.

Using two LED reference spectra would reduce the spectral mismatch errors by a factor of three on average. However, in practical light measurements this would require the end-user to select one of the calibration factors available for example based on estimating the CCT of light being measured. Without carrying a spectrometer, the user would inevitably make selection errors of the calibration factor, losing the theoretical benefit offered by the two reference spectra. This was verified by simulations. Using two reference spectra was discarded at the end, as it did not considerably reduce the spectral errors in practice and would lead to increased complexity and cost of calibrations and measurements. Further tests with the $4103 \mathrm{~K}$ LED reference spectrum showed that it led to the smallest spectral errors also in the case of measuring sources other than LEDs, including daylight (C,D), fluorescent (FL) and high-pressure (HP) discharge lamps (Figure 2, right). For this purpose, the illuminants of [CIE 2004] were used. The calibration source with $4103 \mathrm{~K}$ LED reference spectrum performed best in all cases, except when measuring incandescent light (A) and one type of HP discharge lamp. The detailed results of this spectral analysis have been published in [Kokka 2018]. A proposal for an alternative calculation method for the general mismatch index $f_{1}{ }^{\prime}$, including influence of LED spectra in combination with broadband spectra, has been studied and reported in [FERRERO et. al. 2018].

\subsection{LED standard lamps}

The project has developed new LED standard lamps for luminous intensity and luminous flux, using the LED reference spectrum for selecting the LEDs (Figure 3). The LED standard lamps for luminous intensity, LIS-A, consist of 25 white LEDs assembled on aluminium housing with built-in thermoelectric controller (TEC) and are operated with approximately $65 \mathrm{~mA} \mathrm{DC}$ current, with supply voltage of about $75 \mathrm{~V}$ DC. The luminous intensity level obtained is approximately $245 \mathrm{~cd}$, similar to OSRAM Wi41/G. Two types of luminous flux transfer standard lamps with E27 base have been developed that share the main optical parts, including the diffuse bulb and LEDs. The DC-lamp requires a 15-18 V DC supply voltage and includes a built-in precision current source and a TEC that controls the lamp temperature within $\pm 4{ }^{\circ} \mathrm{C}$ of the set temperature. The second luminous flux standard lamp is aimed at test laboratories who focus on measurement of AC-operated lighting products. The lamp consists of a stable constant power AC/DC-converter operating at $230 \mathrm{~V} \mathrm{AC-voltage.} \mathrm{Both} \mathrm{standard}$ lamps produce approximately $800 \mathrm{Im}$ of luminous flux, and are compatible with laboratories having ambient temperature of $25^{\circ} \mathrm{C}$ [CIE 2015]. 

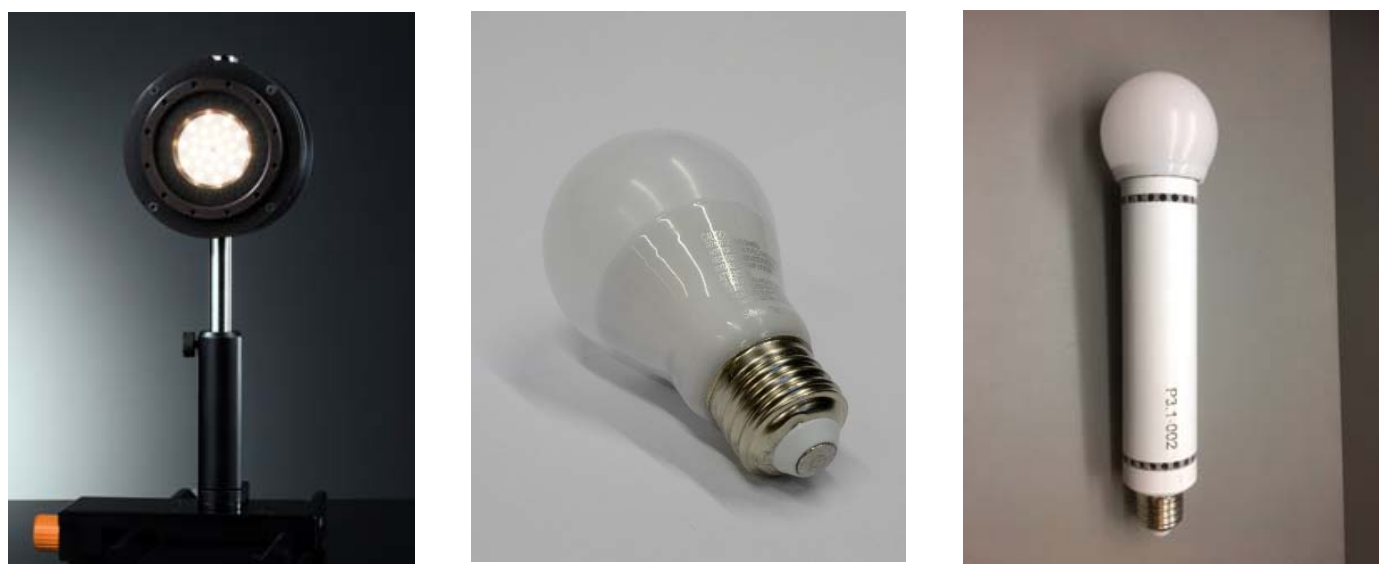

Figure 3 - LED standard lamps developed in the project for DC-operated luminous intensity LIS-A (left), DC-operated luminous flux (centre) and AC-operated luminous flux (right).

\subsection{Unfiltered measurement of white LED illuminance}

The luminous intensity comparison of the project is using, for the first time, a new measurement method, in which the illuminance of the LED standard lamp is measured using unfiltered Predictable Quantum Efficient Detectors (PQEDs) or detectors built using Hamamatsu silicon photodiodes. Both types of detectors are operated with precision apertures, while nitrogen flow through the detectors prevents contamination of the photodiodes. The photometric weighing is obtained numerically by accurate spectral measurement of the LED standard lamp. For this purpose, fully characterised scanning double-monochromator spectroradiometers and array spectroradiometers are used. The unfiltered measurement method is made possible by the naturally bandwidth-limited spectral emission of white LEDs. In the case of the PQED, the method enables measurement of the luminous intensity LED standard lamp directly with a primary standard of optical power. The method is aimed at NMIs for calibration of luminous intensity of LED standard lamps, and for illuminance responsivity of $V(\lambda)$-filtered photometers, integrating sphere photometers and goniophotometers. In addition to the luminous intensity comparison, the unfiltered measurement method is used for the calibration of total luminous flux of the new luminous flux standard lamps, in which the PQED is used for measuring the illuminance of one of the LIS-A standard lamps operated as an external source of the absolute integrating sphere method [Ohno 1998].

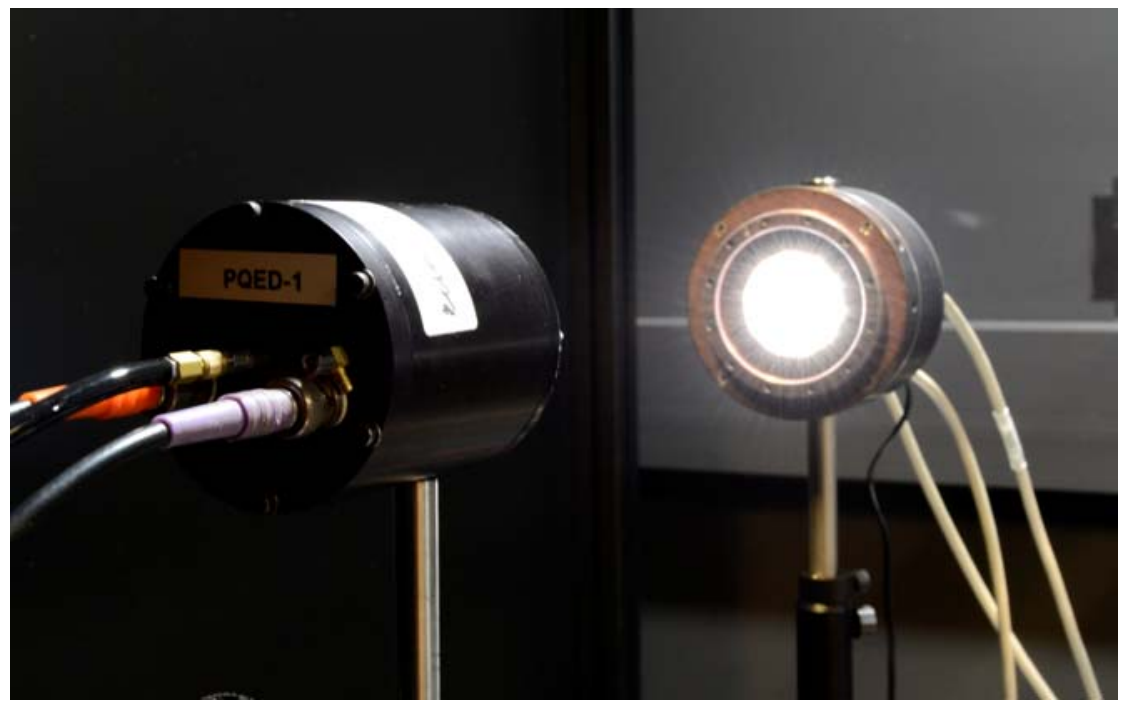

Figure 4 - Illuminance measurement of the luminous intensity LED standard lamp LIS-A using unfiltered Predictable Quantum Efficient Detector (PQED) with nitrogen flow. 


\subsection{Fisheye camera method for integrating spheres}

The project has developed a fast and reliable fisheye camera method for measuring the angular intensity distribution of light sources using a fisheye camera attached to a port of an integrating sphere (Figure 5). The method is primarily aimed at test laboratories for determining spatial nonuniformity correction factors in luminous flux measurements of lighting products, such as LED lamps. Traditionally, applying spatial corrections has required time-consuming goniophotometric measurements. The new method has been validated with 8 integrating sphere photometers and 6 LED lamps, whose angular intensity distributions were measured with 5 goniophotometers for comparison. On average, the relative deviation between the fisheye camera method and goniophotometer method in determining spatial corrections for the artefacts tested was only $0.05 \%$.
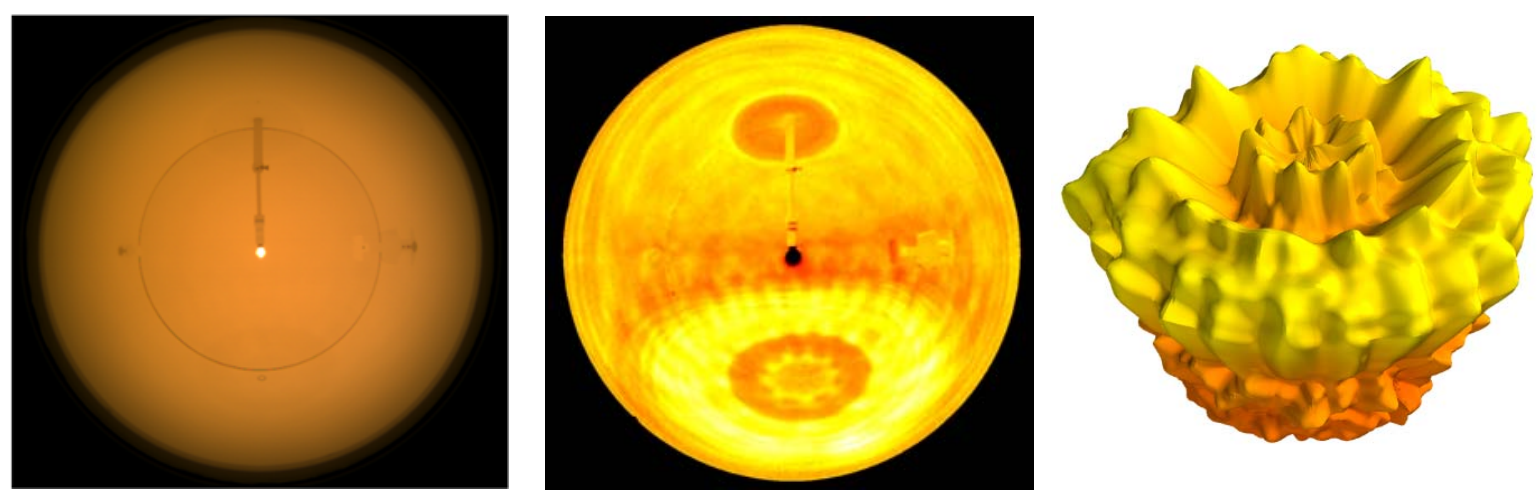

Figure 5 - The fisheye camera method resolves the relative angular intensity distribution of a light source in luminous flux measurement with an integrating sphere. An LED lamp in measurement (left), processed image (centre) and the angular intensity distribution (right).

The fisheye camera method has been described in [Kokka 2017] and the results of the validation with several measurement facilities in [Kokka 2019]. Figure 5 shows a photograph taken with a fisheye camera through a port of an integrating sphere illuminated by an LED lamp under test and the resolved angular intensity distribution of that lamp. The spatial correction factor is calculated using the scanned spatial non-uniformity map of the sphere, a reference image captured for a lamp with known angular intensity distribution, and an image captured for the light source under test. Measuring the angular intensity distribution and spatial nonuniformity correction for a light source takes only a couple of seconds to complete. Another advantage of the measurement method is that it is insensitive to the temporal stability of most light sources, further reducing the time required for the measurements.

\section{Conclusions}

This project has been a unique opportunity, with its partners, collaborators and cooperation with CIE, to carry out scientific research on the fundamental concepts of new LED photometry, including new LED illuminants, LED reference spectrum, LED standard lamps and novel measurement methods that support the specific properties of LED light sources. Current results of the project show that, in the future, photometric calibrations can be carried out using LED standard lamps. The new calibration methods and reference lamp technology solve the potential issue of the current photometric standard lamp types disappearing from the market. The new LED standard lamps provide several advantages, such as improved stability, longer lifetime, robustness for transport and comparisons, as well as reduced spectral errors in measurements of not only LEDs, but other light types as well, including daylight and fluorescent light. The preliminary results of the two photometric comparisons, and experience gained with the new LED standard lamps and measurement methods will be presented in the conference.

\section{Acknowledgements}

The work leading to this study is partly funded by the European Metrology Programme for Innovation and Research (EMPIR) Project 15SIB07 PhotoLED "Future Photometry Based on Solid State Lighting Products". The EMPIR initiative is co-funded by the European Union's 
Horizon 2020 research and innovation programme and the EMPIR Participating States. The project would like to thank CIE and the official collaborators NIM, NRC and IPQ for their support.

\section{References}

CIE 2002. CIE 149:2002. The use of tungsten filament lamps as secondary standard sources. Vienna: CIE.

ISO/CIE 2014. Characterization of the Performance of Illuminance Meters and Luminance Meters. ISO/CIE Standard 19476:2014(E). Vienna: CIE.

CIE 2004. CIE 15:2004. Colorimetry, $3^{\text {rd }}$ Edition. Vienna: CIE.

JOST, S., ET AL., 2017. Determination of illuminants representing typical white light emitting diode sources. Proc. CIE Midterm Meeting 2017, Jeju, Korea.

CIE 2018. CIE 15:2018. Colorimetry, $4^{\text {th }}$ Edition. Vienna: CIE.

KOKKA, A., ET AL., 2018. Development of white LED illuminants for colorimetry and recommendation of white LED reference spectrum for photometry. Metrologia, 55, 526-534.

CIE 2015. CIE standard 025/E:2015. Test Method for LED Lamps, LED Luminaires and LED Modules. Vienna: CIE.

FERRERO, A., ET AL., 2018. Index for the evaluation of the general photometric performance of photometers. Opt. Express 26, 18633-18643.

OHNO, Y., 1998. Detector-based luminous-flux calibration using the Absolute IntegratingSphere Method. Metrologia 35, 473-478.

KOKKA, A., ET AL., 2017. Fisheye camera method for spatial non-uniformity corrections in luminous flux measurements with integrating spheres. Metrologia, 54, 577-583.

KOKKA, A., ET AL., 2019. Validation of the fisheye camera method for spatial non-uniformity corrections in luminous flux measurements with integrating spheres. Metrologia. - In press 